\title{
Study on Mobile Augmented Reality Adoption for Mayo Language Learning
}

\author{
Erasmo Miranda Bojórquez, ${ }^{1}$ Osslan Osiris Vergara Villegas, \\ Vianey Guadalupe Cruz Sánchez, ${ }^{1}$ Jorge Luis García-Alcaraz, ${ }^{2}$ \\ and Jesús Favela Vara ${ }^{3}$

\begin{abstract}
${ }^{1}$ Departamento de Ingeniería Eléctrica y Computación, Universidad Autónoma de Ciudad Juárez, Ciudad Juárez, CHIH, Mexico
${ }^{3}$ Departamento de Ciencias de la Computación, Centro de Investigación Científica y de Educación Superior de Ensenada, Ensenada, BC, Mexico
\end{abstract} \\ ${ }^{2}$ Departamento de Ingeniería Industrial y Manufactura, Universidad Autónoma de Ciudad Juárez, Ciudad Juárez, CHIH, Mexico
}

Correspondence should be addressed to Erasmo Miranda Bojórquez; al132803@alumnos.uacj.mx

Received 20 April 2016; Revised 10 August 2016; Accepted 28 August 2016

Academic Editor: Juan C. Cano

Copyright (C) 2016 Erasmo Miranda Bojórquez et al. This is an open access article distributed under the Creative Commons Attribution License, which permits unrestricted use, distribution, and reproduction in any medium, provided the original work is properly cited.

\begin{abstract}
This paper presents the results of a study applied to undergraduates in order to know how the cultural dimensions affect their perceptions of the acceptance and use of new technologies in a student-centered learning environment. A total of 85 undergraduate students from the Autonomous Indigenous University of Mexico (UAIM) participated in the study. Each student was asked to use a mobile augmented reality (MAR) application designed to learn Mayo language (language spoken in Northwestern Mexico). Afterwards, the students responded to a survey with items concerning the use and technology acceptance and about cultural dimensions of individualism and uncertainty avoidance. Structural equation modeling (SEM) was used to analyze the data collected from students. Results provide evidence that the individualism contributes positively to perceived ease of use of the MAR app, and uncertainty avoidance has no impact. The findings showed that the MAR system could be easily used if it includes a natural way to promote collaborative work. In addition, to gain the trust of students, the uncertainty avoidance needs to be reduced by enriching the help information offered for app use.
\end{abstract}

\section{Introduction}

Progress in transportation technology has facilitated human mobility. The mobility leads to increasing encounters among people with different cultural backgrounds, frequently grouped in different cultural identities. Individuals must have at least two main abilities known as intercultural competence and communication skills to succeed in these encounters [1]. The former refers to the ability to efficiently adapt to the new cultural contexts. The latter refers to the ability to share emotions and create a relationship of effective empathy through the ability to use language.

Intercultural competence is invaluable in educational fields. Schools offer opportunities to develop intercultural competence and communication skills. For example, at university, the undergraduates have the necessity to share information across different cultures and social groups. Therefore, the use of information and communication technologies (ICT), such as augmented reality (AR), could encourage great opportunities for cross-cultural collaborative work in almost all the topics covered in the learning environments [2].

The effectiveness and efficiency of ICT deployment and its use are influenced by national and organizational cultures. During the last decades, culture has been one of the key constructs of research in a number of fields such as finance, learning, public relations, and decision making. In order to understand cultural differences, several models have been developed, such as global leadership and organizational behavior effectiveness (GLOBE) [3], Hofstede et al. [4], and 
Trompenaars and Hampden Turner [5]. There are a number of differences between these models including the time frame of data collection, the type of individuals studied, the number of companies, and the globalization of information. However, Hofstede model was selected in this paper because (a) it is leading cross-cultural research in various disciplines which use quantitative methods; (b) it allows comparing and contrasting the cultural characteristics of individuals against the cultural characteristics of their group, organization, and society; (c) it is flexibile; (d) it allows assessing a number of social trends and phenomena in a systematic way, among others $[6,7]$.

Nowadays, one of the main forms of ICT is the mobile devices. These devices can offer a great quantity of opportunities in the field of education, because they can be used as a learning tool offering students the possibility of capturing real or simulated data and accessing a variety of information anytime and anywhere. According to Khan et al. [8], the type of learning which allows learners to obtain learning materials anywhere and anytime using mobile technologies and the Internet is called mobile learning (m-learning). Mobile devices offer new opportunities to deploy applications aimed at developing intercultural competencies, because they offer portability and ubiquity. According to $[9,10]$, the three main important aspects to use mobile devices in education are (a) portability, (b) instant connectivity, and (c) context sensitivity.

On the other hand, New Media Consortiums 2011 Horizon Report [11] has reported that AR is becoming a technical trend in higher education for making technology blend with virtual and real worlds and is expected to reach mainstream use in education. Current definitions of $A R$ assert that "AR allows a combination of real world elements captured through a camera with multimedia elements such as text, images, video, or 3D models and animations" [12]. For this work, the definition proposed by Azuma [13], who was a pioneer in this topic, was adopted. According to Azuma, AR is a system that fulfills three basic features: (1) a combination of real and virtual worlds, (2) real time interaction, and (3) accurate $3 \mathrm{D}$ registration of virtual and real objects.

In the past, AR applications were created for executing in personal computers. However, with the explosive development of powerful mobile devices, they have become convenient platforms for deploying AR applications, since they include a camera for capturing the real world view, powerful processors to recognize and track the objects of interest, and the capability of rendering and displaying 3D graphics and video. This new area is known as mobile augmented reality (MAR).

An opportunity to perform research that involves the topics mentioned above was detected inside the learning environment of the Autonomous Indigenous University of Mexico (UAIM), located in Northwestern Mexico. Due to its intercultural character, UAIM is focusing on supporting the preservation of indigenous languages. UAIM's main objective is to provide students, with different cultural backgrounds, with a set of student-centered learning tools that preserves their cultural identity. This leads to stimulating students communication skill in their mother language called Mayo and also in Spanish and English. The task of teaching-learning Mayo is typically developed face to face among teachers and students, and great linguistic wealth is involved. However, a problem emerges when the teacher is absent, because the student cannot continue with the learning process. The problem is mainly because the support material for teaching is based on oral traditional media owned by the instructor. Therefore, it could be convenient to design a tool to support students self-learning of Mayo language that can be used everywhere and anywhere, and this can be made by means of a MAR system.

In this paper, a MAR system called Lotería Mayo to support the Mayo language learning is presented. The most important features that system must fulfill are that it needs to be easy of use and take into account the cultural background of the student. Therefore, the technology acceptance model (TAM) extended with the cultural factors of Hofstede model was used to evaluate the MAR system acceptance by 85 students from UAIM.

The rest of the paper is organized as follows. In Section 2 the concepts related to the impact of cultural issues on mobile applications, the use of MAR for learning, and theories about TAM and cultural dimensions are provided. In Section 3 a description of the MAR application is offered. In Section 4 the evaluation of the MAR system is presented, including the objective, the hypotheses, the experimental design, the procedure, the results, and the discussion. Finally, Section 5 presents the conclusions of this research work.

\section{Theoretical Background}

In the following subsections, several important theoretical concepts related to the study presented are briefly explained.

\subsection{The Impact of Cultural Issues on Mobile Applications.} Mobile devices offer new opportunities to deploy applications aimed at developing intercultural competencies, because they offer portability and ubiquity features. Several authors have linked the use of mobile devices with the elements related to culture and technology adoption.

The work proposed in [14] reports on gender patterns identified in a cross-national study of mobile phone use by university students in Sweden, USA, Italy, Japan, and Korea. The set of data was collected from multiple countries to compare the role of gender versus culture (to the extent culture corresponds with nationhood) in use of and attitudes towards mobile telephony. Data were analyzed with respect to the purpose of communication, politeness issues, contact management, and volume of use. Results indicated a number of gendered usage and attitudinal patterns. However, in some cases, cultural variables may prove to be more explanatory than gender.

The study presented in [15] examined the impact of cultural differences on mobile phone adoption patterns. The Hofstedes cultural dimensions were used to examine cultural differences of USA and South Korea, and the Bass diffusion model was used to delineate innovation and imitation effects on mobile phone adoption. The results show that in USA culture innovation factor has a significantly higher level of 
effect on adoption than it does in South Korea culture; and in South Korea culture imitation factor has a higher degree of effect on adoption than it does in USA culture. These findings imply that, in individualistic cultures, people tend to seek information on their own from direct and formal sources, whereas, in collectivistic cultures, people rely more on subjective evaluation of an innovation, conveyed from other like-minded individuals who already have adopted the innovation.

The study presented in [16] examined the current state of the students attitudes towards mobile technology use for second and foreign language learning in higher education. Moreover, the study investigated if age, gender, or cultural factors affect these attitudes. A total of 345 students from two different countries, China (Yunnan University) and Sweden (Dalarna University), participated in the study. To access learners perceptions towards mobile technology use, the Kearneys pedagogical framework to mobile learning from a sociocultural perspective was used. Hofstedes cultural dimensions were used to approach students cultural views. The findings show the respondents attitudes towards mobile learning are very positive with individualization being most positive $(83 \%)$ followed by collaboration (74\%) and authenticity (73\%).

The work in [17] focused on the value consumers can potentially gain from using mobile data services (MDS). The study contributes to the existing body of knowledge about the factors affecting the adoption of MDS, especially in Jordan. A value-based model was developed to investigate the effect of technological, social, and informational influences on mobile value dimensions and the effect of the latter on the adoption intention of MDS. Utilitarian and economic value dimensions are the best predictors of MDS adoption intention in Jordan, and mobile technology forms the strongest influence on people's perception of utilitarian and economic value dimensions. Social and informational influences were also found to be significant but at a lesser degree than technological influences.

In developing countries, especially in Africa, mobile banking can play a strong role, providing a way to overcome financial exclusion and physical distance by allowing local population to conduct financial transactions. An innovative and comprehensive theoretical model that combines the extended unified theory of acceptance and use of technology, with cultural moderators from Hofstede, providing new insights into factors affecting the acceptation and how culture influences individual use behavior was presented in [18]. The model was tested using structural equation modeling (SEM). Performance expectancy, hedonic motivation, and habit were found to be the most significant antecedents of behavior intention. To explain the mobile banking use behavior, the habit and culture moderator effects on behavior intention over use behavior were the most important drivers. Collectivism, uncertainty avoidance, short term, and power distance were found to be the most significant cultural moderators.

The qualitative study in [7] explores the factors responsible for creating economic barriers for 245 women in India, which prevent them from owning a mobile phone. Study findings reveal the specific ways in which cultural factors, like (1) the long power distance between men and women, (2) the gender role defined by Indian society for women, (3) womens attitudes of avoiding uncertainty, and (4) collectivistic practices, create economic barriers for the financially independent study participants. Due to the unfair economic disadvantages generated by the above cultural factors, it becomes challenging for the participants earning a little less than 2 dollars a day to own some of the least expensive mobile phone handsets worth 15 or so on installments of one dollar a month.

As mentioned before, several studies about cultural issues on mobile devices and applications have been presented in the literature; however, the cultural aspect is just beginning to be researched in particular with respect to the acceptance of using mobile technology in learning. Mobile technologies can provide new opportunities to learn a foreign language and include benefits such as flexibility, small size, low cost, and friendly use.

2.2. Mobile Augmented Reality and Its Use for Learning. For the particular case of use of MAR in learning environments, the researchers such as those in [19-21] have suggested that the student can strengthen their learning based on the mixture of the real and the virtual practices. According to the study in [20], MAR can enhance the traditional learning models. The work in [22] explains that the potential power of MAR as a learning tool is its ability to "enable students to see the world around them in new ways and engage with realistic issues in a context with which the students are already connected." In addition, the work presented in [23] shows that MAR is a promising tool for learning because of its ubiquitous availability and computing power offered by mobile devices. However, a challenge remains due to integration problems with traditional learning methods, costs in the development and maintenance of MAR systems, and resistance to the use and acceptance of new technologies.

MAR systems are classified into two types: (a) locationaware and (b) vision-based. The former presents digital media to learners as they move through a physical area with a GPSenabled mobile device. The latter presents the digital media (i.e., text, graphics, audio, video, and 3D models) to learners when they point the camera of a mobile device to certain objects (e.g., QR codes and images) [24].

By all of the above, MAR can be a new way of learning which uses the unique capabilities of mobile devices (flexibility, mobility, and efficiency) in order to offer to the students the opportunity to continue the learning process inside and outside the classroom [25].

\subsection{Technology Acceptance Model (TAM). In 1989, Davis} proposed the TAM which is an information systems theory that models how users come to accept and use a technology [26]. The TAM suggests that the use of a system is a response that can be explained or predicted by the motivation of the user and is directly influenced by an external stimulus of the system features and capabilities [27].

The TAM indicates that perceived ease of use (PEOU) and perceived usefulness (PU) are the two main beliefs that 
determine people intention to use (ITU) technology. PEOU refers to the degree to which a person believes that using a particular system would be free from effort, while PU refers to the degree to which the user believes that a system would improve his or her work performance [28-30]. In the TAM, the degree of technology acceptance is measured by the ITU.

In the literature, there have been several studies about the acceptance and use of technologies particularly focused on mobile devices [16, 25, 27, 31-35]. In most of the works, the TAM was used by adding particular aspects about limitations of the mobile applications and cultural dimensions $[33,36]$. In this paper, the TAM has been used because it is considered as appropriate to predict student satisfaction in learning contexts.

2.4. Culture and Cultural Dimensions. Culture was defined in [6] as the collective programming of the mind which distinguishes the members of one human group from another. Cultural factors are the established beliefs, values, traditions, laws, and languages of a nation or society. The factors are durable and transcending for the development of individuals and they suggest that all these factors can be found behind the different users while using and adopting a particular system [16]. Therefore, it could be of great interest to study learning environments where a mixture of students from diverse cultural backgrounds is included [37].

The study by Hofstede is important because it reveals how people of different countries and cultures interact according to their cultural dimensions. Hofstede provides a values survey measure (VSM 2013) that includes six cultural dimensions to measure and compare different cultures, which are (1) power distance (PD), (2) uncertainty avoidance (UA), (3) individualism versus collectivism (IDV), (4) masculinity versus feminity (MASC), (5) long term orientation versus short-term normative orientation (LTO), and (6) indulgence versus restraint (IVSR) [38].

$\mathrm{PD}$ is "the extent to which a society accepts the fact that power in institutions and organizations is distributed unequally." UA is "the extent to which a society feels threatened by uncertain and ambiguous situations and tries to avoid these situations by providing greater career stability, establishing more formal rules, not tolerating deviant ideas and behaviors, and believing in absolute truths and the attainment of expertise." IDV is "the degree to which people in a country prefer to act as individuals rather than as members of groups." MASC is "the extent to which the dominant values in society are masculine that is, assertiveness, the acquisition of money and things." LTO refers to "future oriented values like persistence and thrift, while a short-term orientation refers to past and present oriented values like respect for tradition and fulfilling social obligations." Finally, IVSR is "the degree to which culture facilitates to regulate the desires and motivation of basic needs."

All the theoretical concepts described provide the basis for the development of the MAR system for learning Mayo language which will expand in the following sections.

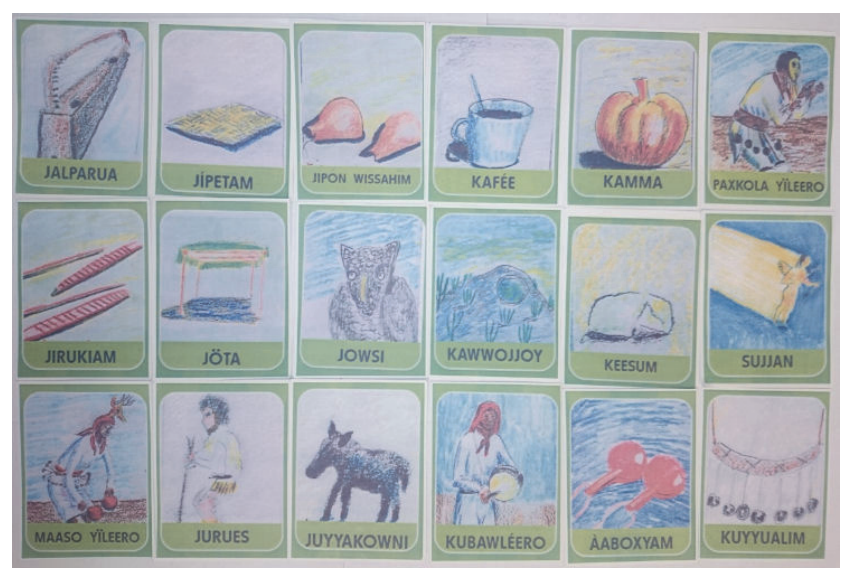

FIgURE 1: Several cards of the deck used as markers.

\section{Lotería Mayo Application}

The application created for this study was called Lotería Mayo. It is based on images and audio using MAR through a card game. The application was inspired by the traditional Mexican game called Lotería (lottery), which is similar to bingo but uses images on a deck of cards instead of numbered ping pong balls. The deck is composed of a set of 89 different images; the drawings in each card represent typical objects, animals, and people from the Mayo social group and were used as markers for the MAR system. All the drawings were provided by professors, in PDF format, who teach the Mayo language at UAIM. An example of several card images is shown in Figure 1.

Before the start of each game, players choose what table (tabla) they want to play with. The table (shown in Figure 2) is a board with a randomly created $4 \times 4$ grid of pictures with their corresponding name. The game starts with the cantor randomly selecting a card and showing it to the camera of the mobile device; immediately, the process of recognizing an object starts by comparing the extracted features against the database of 89 objects previously defined as image targets. If a known object is recognized, then the real model is superimposed in the real scene, the corresponding audio with the pronunciation of the Mayo word is reproduced followed by an explanation in Spanish language, and the process of tracking is initiated. This process is repeated every time the camera points at a card which means that the student can repeat this process to learn. If the image of the card appears on the player table, the player marks the corresponding image with small rocks or beans. The process is repeated until the first player completes all the images in the table and shouts Lotería! An example of augmentation results can be observed in Figure 3.

The system was developed with Unity and Vuforia platforms, and the camera of the mobile device was used to recognize the card drawings. The MAR system was initially installed for tests, on a laptop with Windows 7 operating system. Subsequently, the system was installed on a Sony Xperia $\mathrm{Z1}$, on Asus and Samsung Galaxy tablets. The application can 


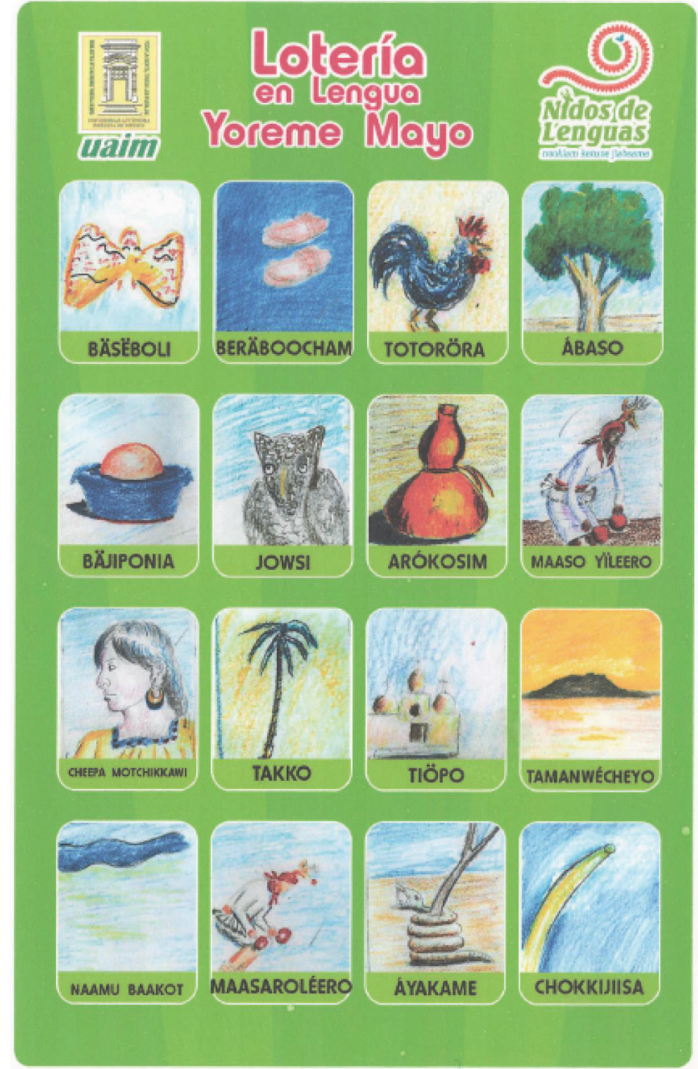

FigURE 2: An example of one board (table) for lottery game.

be executed on devices with Android operating system 4.2.1 and up.

Unity, that is, a multiplatform game engine created by Unity Technologies, was selected because of the facility of having control of the content of the mobile device in a local way. The visual environment of the platform provides integration with Vuforia that allows the creation of AR content, including the most important operations such as object recognition and tracking. The drawings of each card were defined in Vuforia as image targets and renamed using their respective word in Mayo language for easy identification. Then, a test to observe the capability of recognition for each object was executed, obtaining a great success. At the end, a digital image texture representing the real object for each drawing and its corresponding audio in $\mathrm{mp} 3$ format was assigned to each image target plane. An example of the real models superimposed on the card drawings is shown in Figure 4.

\section{Evaluation of Lotería Mayo}

In order to explore the potentials and limitations of the system, a study was performed to assess the PEOU and PU that influence the ITUMAR system.

4.1. Objective of the Evaluation. The aim of the study was to assess the MAR system through variables related to the perception and the cultural dimensions of the undergraduates to predict the ITUMAR system. The study is relevant because it will allow the process of system redesign including the characteristics of PEOU, PU, and culture of the students. This could increase the probability that the MAR system will be used inside the learning environments.

4.2. Research Hypothesis Development. The TAM was originally designed to provide an explanation of user behavior when using information systems [28]. An objective of TAM is to establish the basis for tracing the impact of external factors, internal beliefs, attitudes, and intentions of users. The ITUMAR system is determined by PEOU and PU of the user. Therefore, the TAM can be used to identify the reasons why a system is unacceptable by the user and as a consequence design the appropriate corrective steps.

In this study, the TAM was used to observe the perceptions of undergraduates in the task of learning the Mayo language through a MAR system. The proposed model is an extension of TAM due to the addition of two cultural dimensions extracted from Hofstede model [38].

According to TAM, the PU and the PEOU affect the student intention to adopt a new technology. In fact, the less effort the students expect to invest in using the technology, the more useful they perceive it to be [39]. Therefore, the following three hypothesis can be postulated:

(H1) Students PU of MAR will positively affect their ITUMAR system.

(H2) Students PEOU of MAR will positively affect their ITUMAR system.

(H3) Students PEOU of MAR will positively affect PU of the MAR system.

According to Hofstede et al. [4], culture is specific to the group or society and there is no absolute measure for evaluating it. Therefore, culture is described by external observers based on the manifestations of common practices and values. The common practices are composed of symbols, heroes, and rituals, and the values are considered as the core of the culture that is acquired and developed at an early age. Hofstede et al. refer to the set of culture values as the national value system. Hofstede's cultural dimensions are the tools used to measure the acceptance and use of MAR system according to cultural diversity of undergraduates [37]. In this study, the cultural dimensions of IDV and UA were selected, because of the conditions of the learning environment at the UAIM. In the daily environment of UAIM undergraduates, the students work individually or collectively faced with uncertainty of the new technologies. Therefore, according to $[6,15,16,40]$, two hypotheses associated with cultural factors can be postulated:

(H4) Students IDV will positively affect their PEOU of the MAR system.

(H5) Students UA will positively affect their PEOU of the MAR system.

4.3. Experimental Design and Procedure. Once the hypotheses have been established, the proposed extended TAM can 


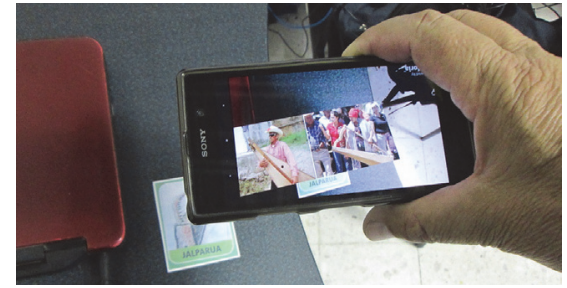

(a)

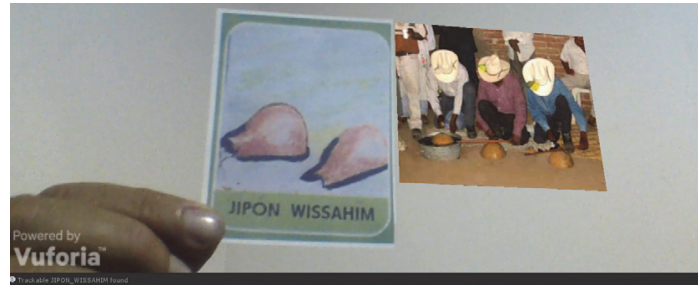

(b)

FIGURE 3: Example of augmentation obtained with Lotería Mayo: (a) with a mobile device and (b) with a laptop.

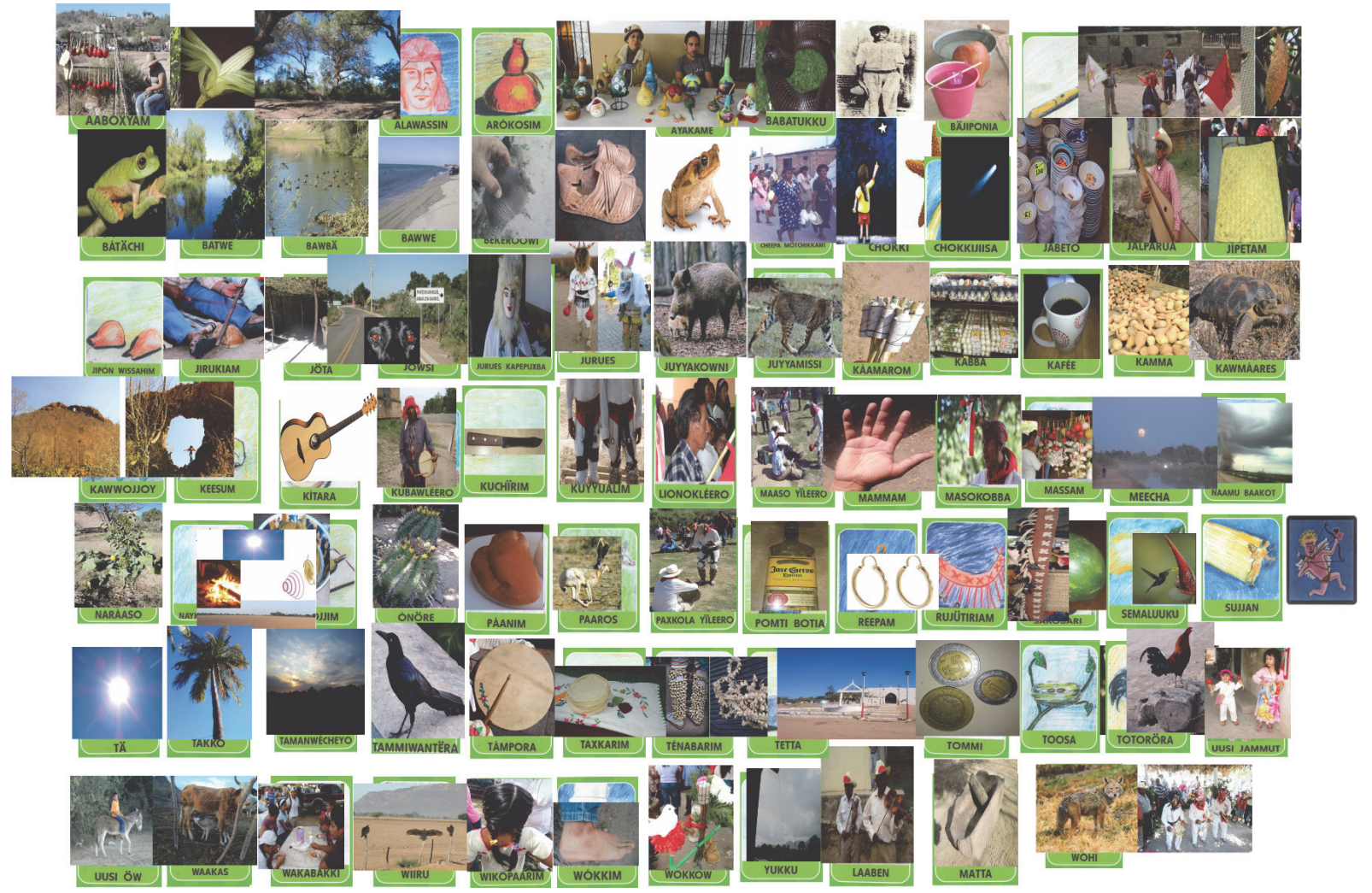

FIgURE 4: The real models superimposed on the plane of card drawings.

be defined as shown in Figure 5. The opportunity of students to continue the learning process inside and outside the classroom can be improved and become more efficient by including the characteristics obtained from the results of the survey applied related to TAM, which predicts the ease of use offered by the MAR system. Another factor that also influences the use of the MAR system is the set of behaviors and languages learned by the student within his social group (cultural dimensions). The justification for relating IDV and UA with the latent variable PEOU has two assumptions: (1) the ability to take advantage of what students have learned to work collectively inside their social group and (2) the proper handling of uncertainty to new technologies, facilitating the use of MAR system through strategies such as collaborative or group work. Both assumptions can happen in the Lotería game.
The study was performed at classrooms of UAIM, located in Los Mochis, Sinaloa, Mexico. The sample consisted of a total of 85 indigenous undergraduates from different levels and academic programs, and a within-subjects design was used.

The session to perform the study consisted of an explanation offered to undergraduates about the goal of the research and the corresponding instructions to manipulate the MAR application. Afterwards, the students randomly selected ten cards from the deck of 89 cards and started the application use. Three tablets were used; thus 3 students could simultaneously test the application. After that, another three students would do the same; the process was repeated until all the students had a chance to test the application. The average time spent by each student was 5 minutes. Finally, with the goal of examining the hypothesis, a printed survey was filled 


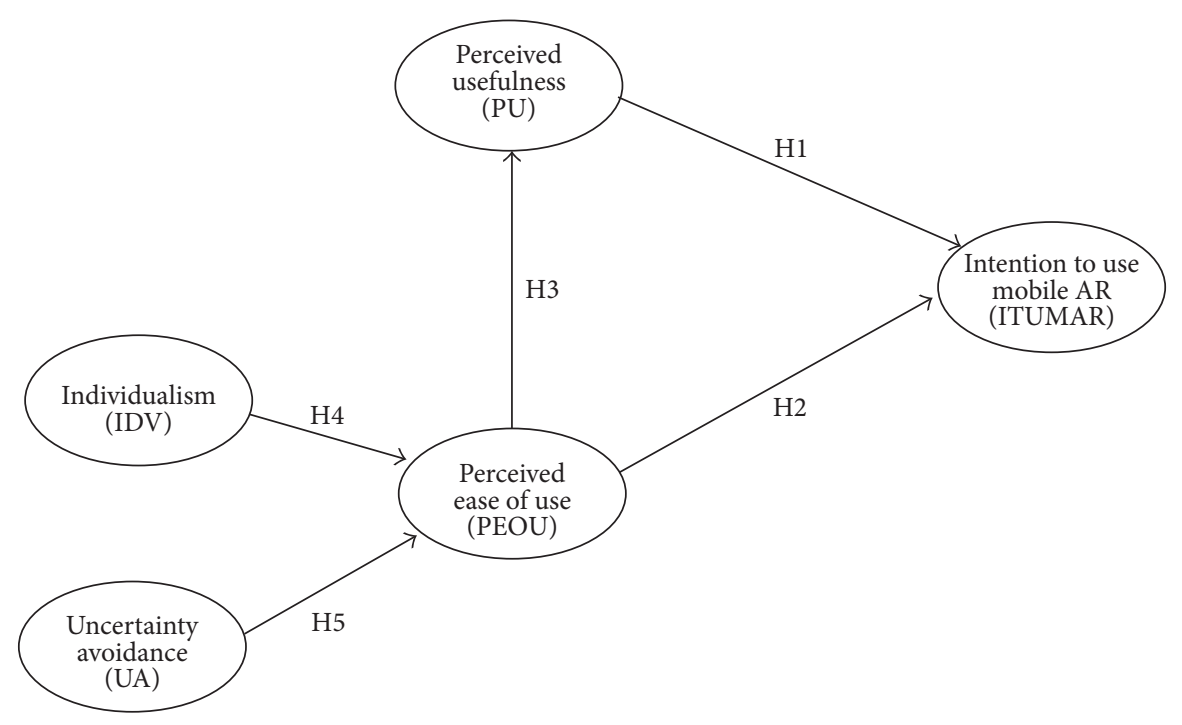

FIGURE 5: The proposed research model.

in by each student in order to know their perception of the use of the MAR system. Data collection took place during the first week of July 2015.

The survey (shown in the Appendix) was designed and structured in three main sections. The first one is composed of three questions to collect student personal data such as age, gender, and ethnicity. The second one includes 9 statements regarding the PEOU, PU, and ITUMAR for learning the Mayo language, adapted from English version of "VSM 2013 questionnaire" [38]. Finally, the third one includes 8 items related to TAM cultural dimensions of IDV and UA.

The constructs were valued based on 4 different sevenpoint Likert scales: Scale A corresponds to the following: 1 Strongly disagree, 2 In disagreement, 3 Somewhat disagree, 4 I do not know, 5 Somewhat agree, 6 In agreement, 7 Strongly agree. Scale B corresponds to the following: 1 Strongly unimportant, 2 Without unimportant, 3 Somewhat unimportant, 4 I do not know, 5 Somewhat important, 6 Important, 7 Very important. Scale C corresponds to the following: 7 Very good, 6 Good, 5 Little good, 4 Fair, 3 Little poor, 2 Poor, 1 Very poor. And Scale D corresponds to the following: 1 Usually, 2 Sometimes, 3 Little, 4 I do not know, 5 It is rare, 6 Seldom, 7 Never. The latent variables with their corresponding indicators and scales are depicted in Table 1.

The data analysis of this study was based on a SEM approach, because it can help to represent the relationships among the latent variables. The SEM has been proven to allow the simultaneous analysis for assessing the relationships among variables, and the errors for each variable can be independently estimated [41]. The structural model (path model) that visually depicts the hypotheses and the latent variables with their corresponding indicators is shown in Figure 6 . The indicators locked into rectangles correspond to the statements of the survey.

The SmartPLS 3 software, that is, a tool for partial least squares structural equation modeling (PLS-SEM), was used to examine the relationships between indicators and to
TABLE 1: Latent variables indicators with their corresponding Likert scales.

\begin{tabular}{llcccc}
\hline Indicators & PEOU & PU & ITUMAR & IDV & UA \\
\hline PU1 & Scale A & & & & \\
PU2 & Scale A & & & & \\
PU3 & Scale A & & & & \\
PU4 & Scale A & & & & \\
PEOU1 & & Scale A & & & \\
PEOU2 & & Scale A & & & \\
PEOU3 & & Scale A & & & \\
ITUMAR1 & & & Scale A & & \\
ITUMAR2 & & & Scale A & & \\
CF1 & & & & Scale B & \\
CF2 & & & & Scale B & \\
CF3 & & & & Scale B & \\
CF4 & & & & Scale B & \\
CF5 & & & & & Scale C \\
CF6 & & & & Scale D \\
CF7 & & & & Scale A \\
CF8 & & & &
\end{tabular}

confirm if the proposed hypotheses were valid. Before analyzing and discussing the SEM, the reliability and validity of the measurement model were checked through the indicators. It is necessary to determine whether the measures of the data have satisfactory properties such as internal consistency reliability, convergent validity, and discriminant validity.

4.3.1. Internal Consistency Reliability. The goal is to obtain a measure based on the correlations among different items of the same instrument. It is important to measure whether several items proposed to measure the same general construct produce similar scores. Cronbach's alpha $(\alpha)$ quantifies the internal consistency of the data through the degree to which 


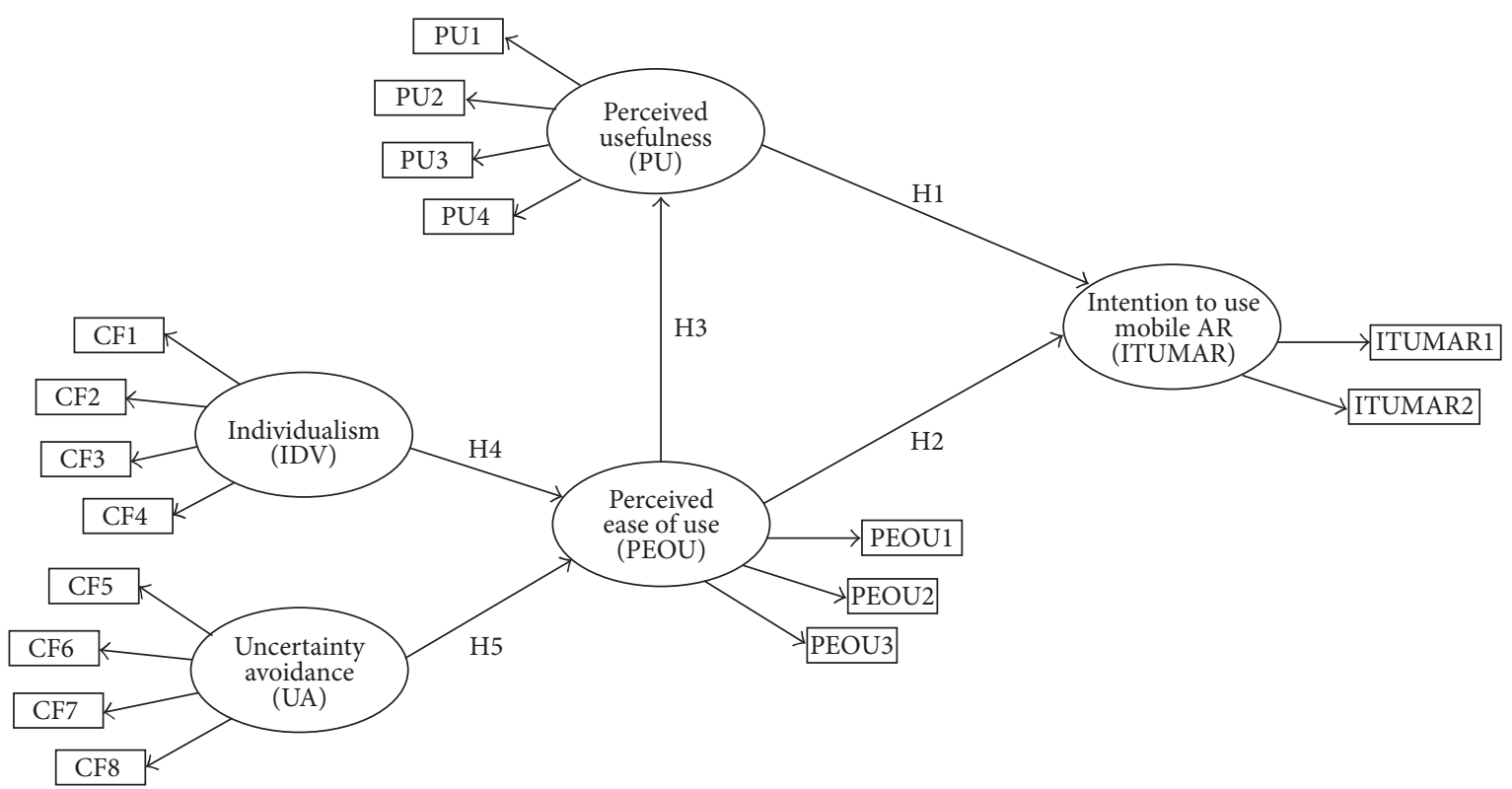

FIgURE 6: Structural model with paths, latent variables, and indicators.

the items on an instrument are correlated. $\alpha$ normally ranges between 0 and 1 , where values from 0.0 to 0.40 are considered low, from 0.40 to 0.60 are considered moderate, from 0.60 to 0.80 are considered good, and from 0.80 to 1.0 are considered high.

4.3.2. Convergent Validity. The convergent validity can be established by showing that measures that should be related are in reality related. Convergent validity is the degree to which a measure correlates positively with the alternative measures of the same construct. The items that are indicators (measures) of a specific construct should converge or share a high proportion of variance. Therefore, to measure the convergent validity, the average variance extracted (AVE) was used.

The AVE is the average amount of variation that a latent construct is able to explain in the observed variables to which it is theoretically related. It is considered adequate when its value is $\geq 0.5$; this indicates that, on average, a construct explains more than half of the variance of its indicators.

4.3.3. Discriminant Validity. Discriminant validity is the extent to which a construct is truly distinct from other constructs by empirical standards. This implies that a construct is unique and captures phenomena not represented by other constructs in the model. Two measures of discriminant validity were used in this study, the Fornell-Larcker criterion and the cross loadings [42].

The Fornell-Larcker criterion suggests that discriminant validity is established if a latent variable accounts for more variance in its associated indicator variables than it shares with other constructs in the same model. To satisfy this requirement, the square root of the AVE of each construct should be greater than its highest correlation with any other construct. On the other hand, the cross (outer) loading of an indicator on the associated construct should be greater than all of its loadings on other constructs.

4.4. Results. From the total sample of 85 students, $49 \%$ (42) were male and $51 \%$ (43) were female. The predominant age of the students comprises the following: $45 \%$ (38) ranged between 20 and 24 years, 24\% (20) between 25 and 29 years, and $11 \%$ (9) between 30 and 34 years. Regarding the question of membership to an ethnic group, 12\% (10) of students responded with yes and $88 \%$ (75) said no. The corresponding values of $\alpha$, mean, median, and standard deviation for the latent variables of the proposed SEM are shown in Table 2.

As can be observed in Table 2, $\alpha$ values for this research ranged from 0.701 to 0.921 ; therefore, the reliability of data is valid. Furthermore, the internal consistency of the latent variables oscillates between good and high values, which were ranged from 0.60 to 1.0. The computed values of AVE for each latent variable are shown in Table 3.

Table 3 shows that all the AVE values are higher than 0.5 , which defines a positive correlation and as a consequence convergent validity. The Fornell-Larcker values computed for the proposed model are shown in Table 4, and the cross loadings values are shown in Table 5.

As Table 4 demonstrates, the AVE square root for each construct is greater than its shared variance with any other construct. Furthermore, as can be observed from Table 5, the outer loadings of a construct are higher than all its cross loadings with other constructs; therefore discriminant validity is supported. Once confirming that the data in the indicators and variables were reliable and valid, the next step was to evaluate the results of the SEM. This involves examining the model predictive capabilities and the relationships between the constructs. For this, the significant level of the path coefficients, the coefficient of determination $\left(R^{2}\right)$, and the total effects of each latent variable were computed. 
TABLE 2: Descriptive statistics of indicators.

\begin{tabular}{|c|c|c|c|c|}
\hline Latent variables & Mean & Median & Std. dev. & $\alpha$ \\
\hline \multicolumn{5}{|l|}{ TAM variables } \\
\hline Perceived usefulness (PU) & & & & 0.921 \\
\hline PU1 & 6.14 & 7 & 1.21 & \\
\hline PU2 & 6.19 & 6 & 0.98 & \\
\hline PU3 & 6.07 & 6 & 1.14 & \\
\hline PU4 & 6.16 & 7 & 1.24 & \\
\hline Perceived ease of use (PEOU) & & & & 0.908 \\
\hline PEOU1 & 5.89 & 6 & 1.21 & \\
\hline PEOU2 & 6.21 & 6 & 1.18 & \\
\hline PEOU3 & 6.14 & 7 & 1.38 & \\
\hline Intention to use the MAR (ITUMAR) & & & & 0.701 \\
\hline ITUMAR1 & 6.13 & 6 & 1.33 & \\
\hline ITUMAR2 & 6.18 & 6 & 1.12 & \\
\hline \multicolumn{5}{|l|}{ Cultural factors (CF) } \\
\hline Individualism (IDV) & & & & 0.919 \\
\hline CF1 & 6.65 & 7 & 0.92 & \\
\hline CF2 & 6.69 & 7 & 1.02 & \\
\hline CF3 & 6.69 & 7 & 1.08 & \\
\hline CF4 & 6.58 & 7 & 1.29 & \\
\hline Uncertainty avoidance (UA) & & & & 0.840 \\
\hline CF5 & 6.38 & 6 & 1.48 & \\
\hline CF6 & 4.12 & 5 & 2.14 & \\
\hline CF7 & 4.22 & 5 & 2.54 & \\
\hline CF8 & 5.25 & 6 & 2.58 & \\
\hline
\end{tabular}

TABLE 3: AVE values for latent variables of the proposed model.

\begin{tabular}{ll}
\hline Latent variables & AVE \\
\hline ITUMAR & 0.765 \\
IDV & 0.799 \\
PU & 0.805 \\
PEOU & 0.846 \\
UA & 0.590 \\
\hline
\end{tabular}

TABLE 4: The Fornell-Larcker values of the latent variables of the proposed model.

\begin{tabular}{lccccc}
\hline Latent variables & IDV & ITUMAR & PU & PEOU & UA \\
\hline IDV & $\mathbf{0 . 8 9 4}$ & & & & \\
ITUMAR & 0.655 & $\mathbf{0 . 8 7 5}$ & & & \\
PU & 0.212 & 0.599 & $\mathbf{0 . 8 9 7}$ & & \\
PEOU & 0.452 & 0.769 & 0.742 & $\mathbf{0 . 9 2 0}$ & \\
UA & 0.776 & 0.530 & 0.037 & 0.276 & $\mathbf{0 . 7 6 8}$
\end{tabular}

The estimation of path coefficients in SEM is based on ordinary least squares (OLS) regressions of each endogenous latent variable on its corresponding predecessor constructs. The path coefficients may be biased if the estimation involves significant levels of collinearity among the predictor constructs [41]. Therefore, it is important to measure the level of collinearity inside the model. Two measures were computed
TABLE 5: Results from the cross loadings computation for the proposed model.

\begin{tabular}{lccccc}
\hline \multirow{2}{*}{ Indicators } & \multicolumn{5}{c}{ Latent variables } \\
& IDV & ITUMAR & PU & PEOU & UA \\
\hline CF1 & $\mathbf{0 . 9 2}$ & 0.60 & 0.27 & 0.51 & 0.67 \\
CF2 & $\mathbf{0 . 9 5}$ & 0.60 & 0.21 & 0.37 & 0.71 \\
CF3 & $\mathbf{0 . 9 1}$ & 0.65 & 0.19 & 0.42 & 0.75 \\
CF4 & $\mathbf{0 . 7 9}$ & 0.41 & -0.10 & 0.16 & 0.72 \\
CF5 & 0.76 & 0.49 & 0.00 & 0.26 & $\mathbf{0 . 9 2}$ \\
CF6 & 0.64 & 0.48 & 0.08 & 0.25 & $\mathbf{0 . 9 1}$ \\
CF7 & 0.56 & 0.22 & -0.14 & -0.02 & $\mathbf{0 . 6 3}$ \\
CF8 & 0.54 & 0.19 & -0.26 & 0.02 & $\mathbf{0 . 5 4}$ \\
ITUMAR1 & 0.53 & $\mathbf{0 . 9 2}$ & 0.64 & 0.77 & 0.39 \\
ITUMAR2 & 0.64 & $\mathbf{0 . 8 3}$ & 0.38 & 0.55 & 0.58 \\
PEOU1 & 0.35 & 0.65 & 0.70 & $\mathbf{0 . 8 7}$ & 0.25 \\
PEOU2 & 0.41 & 0.69 & 0.67 & $\mathbf{0 . 9 3}$ & 0.21 \\
PEOU3 & 0.49 & 0.77 & 0.68 & $\mathbf{0 . 9 5}$ & 0.30 \\
PU1 & 0.10 & 0.44 & $\mathbf{0 . 9 1}$ & 0.57 & -0.10 \\
PU2 & 0.06 & 0.38 & $\mathbf{0 . 8 7}$ & 0.55 & -0.02 \\
PU3 & 0.27 & 0.56 & $\mathbf{0 . 9 2}$ & 0.67 & 0.07 \\
PU4 & 0.27 & 0.69 & $\mathbf{0 . 8 9}$ & 0.80 & 0.13 \\
\hline
\end{tabular}

to do this: the tolerance (TOL) and the variance inflation factor (VIF). 
TABLE 6: Results obtained for TOL and VIF.

\begin{tabular}{lccc}
\hline Latent variables & Indicators & TOL & VIF \\
\hline \multirow{3}{*}{ PU } & PU1 & $\mathbf{0 . 1 6 2}$ & 4.014 \\
& PU2 & 0.249 & 3.175 \\
& PU3 & 0.222 & 3.713 \\
& PU4 & $\mathbf{0 . 1 7 3}$ & 2.395 \\
\multirow{2}{*}{ PEOU } & PEOU1 & 0.262 & 2.201 \\
& PEOU2 & $\mathbf{0 . 1 5 6}$ & 4.639 \\
\multirow{2}{*}{ ITUMAR } & PEOU3 & $\mathbf{0 . 1 2 6}$ & $\mathbf{5 . 3 5 6}$ \\
& ITUMAR1 & 0.251 & 1.411 \\
& ITUMAR2 & 0.376 & 1.411 \\
\multirow{2}{*}{ IDV } & CF1 & $\mathbf{0 . 1 9 0}$ & 3.356 \\
& CF2 & $\mathbf{0 . 1 5 8}$ & $\mathbf{5 . 1 7 9}$ \\
& CF3 & $\mathbf{0 . 1 7 4}$ & 3.555 \\
& CF4 & 0.283 & 2.224 \\
\hline \multirow{2}{*}{ UA } & CF5 & 0.283 & 1.980 \\
& CF6 & 0.345 & 2.123 \\
& CF7 & 0.397 & 2.112 \\
& CF8 & 0.440 & 1.696 \\
\hline
\end{tabular}

The TOL represents the amount of variance of one formative indicator not explained by the other indicators in the same block. The VIF is a measure of the degree of multicollinearity among the latent variables that are hypothesized to affect another latent variable. It measures how much the variance of an estimated regression coefficient is increased (inflated) because of collinearity. A TOL value of 0.20 or lower and a VIF value of 5 and higher, respectively, indicate a potentially harmful multicollinearity problem. The software statistical package for the social sciences (SPSS) was used to compute the TOL and VIF values of the proposed model; the results obtained are shown in Table 6.

According to VIF values in Table 6, there are no critical levels of collinearity, except for two indicators: PEOU3 and CF2. Moreover, the TOL values for 7 of the 17 variables are not $\geq 0.2$; however, 15 variables are $<5$ for VIF. Therefore, the multicollinearity problem does not need to be solved.

The PLS-SEM algorithm applies partial regression models to estimate and represent the hypothesized relationships between the latent variables [41]. The estimated path coefficients values range between -1 and +1 . Coefficients close to +1 represent strong positive relationships, whereas strong negative relationships are defined when the values are close to -1 . The $R^{2}$ coefficient is a measure of the predictive accuracy of the model; it took into account the weight of each indicator to identify which specific element should be addressed to suggest positive action. The corresponding values between relationships and $R^{2}$ are shown in Figure 7.

The significance level of the path coefficients can be found in two ways: the first one consists in comparing a critical value against a value $t$ of theoretical student; the second one consists in verifying whether within the limits of the intervals of confidence the zero value is included. The significance level of a path depends on the standard error coefficient for computing the critical value of $t$ compared with the theoretical value of $t=1.96$ with an error of $5 \%$ for a twotailed $t$-test.

In addition to computation of $t$ values, several researchers report the $p$ value to measure the significant level of a path. PLS-SEM assumes [41] that the data are not normally distributed; this involves using a nonparametric bootstrap procedure to test if the outer weights, the outer loadings, and the path coefficients are significant in the analysis. The bootstrapping routine uses 500 samples with random coefficient values to achieve a distribution that can be seen as a reasonable approximation of the distribution estimated coefficients, and the standard deviation can be used to calculate the standard error through software. The computed values for $t$, standard error, confidence intervals, $p$ of latent variables, and significance level are shown in Table 7.

From Table 7, the limits of the confidence interval variable latent paths for IDV and PEOU were calculated as follows: the lower limit: $0.599-1.96 * 0.260=0.089$, and the upper limit: $0.599+1.96 * 0.260=1.108$. The value of 0.599 corresponds to the original value of the coefficient. 1.96 is the critical value of $t$ for an error of $5 \%$, and the value of 0.260 is the standard error. The values of the outer weights for each indicator are shown in Table 8, whereas the indirect and total effects of latent variables are shown in Tables 9 and 10, respectively.

Finally, according to Hofstede VSM 2013 manual, the cultural factors were also calculated. The IDV index was obtained with

$$
\mathrm{IDV}=35(\mathrm{CF} 2-\mathrm{CF} 1)+35(\mathrm{CF} 4-\mathrm{CF} 3)+C(i c) .
$$

The UA index is obtained with

$$
\mathrm{UAI}=40(\mathrm{CF} 5-\mathrm{CF} 6)+25(\mathrm{CF} 7-\mathrm{CF} 8)+C(u a) .
$$

To calculate (1) and (2), the averages of each indicator must be computed. $C(i c)$ and $C(u a)$ are positive or negative constants used to ensure that the index is inside the range of $0-100$. The indices obtained for students of the UAIM are shown in Table 11.

4.5. Discussion. The sample size of the study was 85 students, and the latent variables used were UA, IDV, PEOU, PU, and ITUMAR. After the analysis of the data collected, seventeen indicators were obtained: 8 related to cultural factors ( 4 for UA and 4 for IDV), 4 related to PU, 3 related to PEOU, and 2 related to ITUMAR.

The characteristics of the data such as sample size, nonnormal, and the use of different measurement scales, were the main reasons to use PLS-SEM in this study. PLS-SEM works efficiently with small samples size and complex models, because it reaches high levels of statistical power with the samples available $[41,43,44]$. The work in [45] presents the criteria to take into account to establish the minimum size of the sample to have valid data by means of PLS-SEM. A synthesized version of the criteria established by Cohen, in a form of table, can be found in [41], and it was used in this study. According to the five independent variables, a minimum value to detect $R^{2}$ of 0.25 and an estimation of $5 \%$ error probability were selected. As a result, Hair et al. suggest 


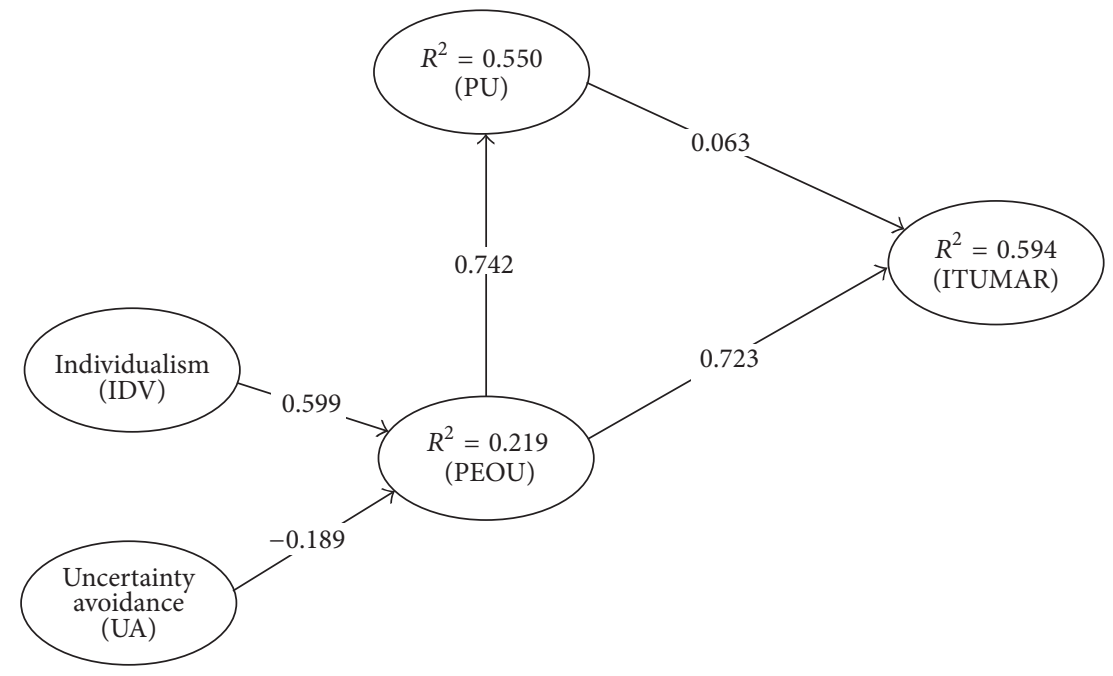

FIGURE 7: $R^{2}$ and path coefficients of the proposed SEM.

TABLE 7: Path coefficients of the SEM proposed.

\begin{tabular}{lcccccc}
\hline Latent variables paths & Path coefficients & $t$ & Standard error & Significance level & $p$ & Confidence intervals \\
\hline IDV $\rightarrow$ PEOU & 0.599 & 2.304 & 0.260 & $* * *$ & 0.022 & {$[0.089,1.108]$} \\
PU $\rightarrow$ ITUMAR & 0.063 & 0.289 & 0.219 & NS & 0.773 & {$[-0.366,0.493]$} \\
PEOU $\rightarrow$ ITUMAR & 0.723 & 3.752 & 0.193 & $* * *$ & 0.000 & {$[0.345,1.100]$} \\
PEOU $\rightarrow$ PU & 0.742 & 9.096 & 0.082 & $* * *$ & 0.000 & {$[0.582,0.902]$} \\
$\mathrm{UA} \rightarrow$ PEOU & -0.189 & 0.873 & 0.216 & NS & 0.383 & {$[-0.613,0.235]$} \\
\hline
\end{tabular}

${ }^{* * *}$ Significance level of $5 \%$.

NS: no significance.

TABLE 8: Outer weights significance testing results.

\begin{tabular}{lccccccc}
\hline Latent variables & Indicators & Outer weights & $t$ & Standard error & Significance level & $p$ & Confidence intervals \\
\hline \multirow{3}{*}{ IDV } & CF1 & $0.384(0.923)$ & 2.618 & 0.147 & $* * *$ & 0.009 & {$[0.096,0.671]$} \\
& CF2 & $0.281(0.945)$ & 3.909 & 0.072 & $* * *$ & 0.000 & {$[0.140,0.422]$} \\
& CF3 & $0.315(0.908)$ & 3.360 & 0.094 & $* * *$ & 0.001 & {$[0.131,0.498]$} \\
\hline \multirow{4}{*}{ UA } & CF4 & $0.120(0.791)$ & 0.705 & 0.170 & NS & 0.481 & {$[-0.214,0.454]$} \\
& CF5 & $0.555(0.916)$ & 1.616 & 0.344 & NS & 0.107 & {$[-0.118,1.229]$} \\
& CF6 & $0.536(0.911)$ & 1.518 & 0.353 & NS & 0.130 & {$[-0.156,1.227]$} \\
\hline \multirow{2}{*}{ ITUMAR } & CF7 & $-0.035(0.633)$ & 0.105 & 0.332 & NS & 0.917 & {$[-0.686,0.616]$} \\
& CF8 & $0.048(0.538)$ & 0.202 & 0.239 & NS & 0.840 & {$[-0.421,0.517]$} \\
\hline \multirow{3}{*}{ PEOU } & ITUMAR1 & $0.667(0.919)$ & 7.994 & 0.083 & $* * *$ & 0.000 & {$[0.503,0.830]$} \\
& ITUMAR2 & $0.468(0.828)$ & 8.104 & 0.058 & $* * *$ & 0.000 & {$[0.355,0.581]$} \\
\hline \multirow{4}{*}{ PU } & PEOU1 & $0.343(0.871)$ & 18.523 & 0.019 & $* * *$ & 0.000 & {$[0.307,0.380]$} \\
& PEOU2 & $0.357(0.933)$ & 26.714 & 0.013 & $* * *$ & 0.000 & {$[0.331,0.383]$} \\
& PEOU3 & $0.385(0.954)$ & 19.149 & 0.020 & $* * *$ & 0.000 & {$[0.346,0.425]$} \\
\hline
\end{tabular}

*** Significance level of $5 \%$.

NS: no significance. 
TABLE 9: Indirect effects of latent variables.

\begin{tabular}{|c|c|c|c|c|}
\hline Latent variables & Indirect effects & $t$ & Significance levels & $p$ \\
\hline IDV $\rightarrow$ ITUMAR & 0.461 & 2.199 & $* * *$ & 0.028 \\
\hline $\mathrm{IDV} \rightarrow \mathrm{PU}$ & 0.444 & 2.422 & $* * *$ & 0.016 \\
\hline $\mathrm{IDV} \rightarrow \mathrm{PEOU}$ & - & - & - & - \\
\hline $\mathrm{PU} \rightarrow \mathrm{ITUMAR}$ & - & - & - & - \\
\hline PEOU $\rightarrow$ ITUMAR & 0.047 & 0.278 & NS & 0.781 \\
\hline $\mathrm{PEOU} \rightarrow \mathrm{PU}$ & - & - & - & - \\
\hline $\mathrm{UA} \rightarrow$ ITUMAR & -0.145 & 0.851 & NS & 0.395 \\
\hline $\mathrm{UA} \rightarrow \mathrm{PU}$ & -0.140 & 0.890 & NS & 0.374 \\
\hline $\mathrm{UA} \rightarrow \mathrm{PEOU}$ & - & - & - & - \\
\hline
\end{tabular}

TABLE 10: Total effects of latent variables.

\begin{tabular}{|c|c|c|c|c|c|}
\hline Latent variables & Total effects & $t$ & Significance levels & $p$ & 95\% confidence intervals \\
\hline IDV $\rightarrow$ ITUMAR & 0.461 & 2.199 & $* * *$ & 0.028 & {$[0.050,0.871]$} \\
\hline $\mathrm{IDV} \rightarrow \mathrm{PU}$ & 0.444 & 2.422 & $* * *$ & 0.016 & {$[0.085,0.804]$} \\
\hline $\mathrm{IDV} \rightarrow \mathrm{PEOU}$ & 0.599 & 2.304 & $* * *$ & 0.022 & {$[0.089,1.108]$} \\
\hline $\mathrm{PU} \rightarrow \mathrm{ITUMAR}$ & 0.063 & 0.289 & NS & 0.773 & {$[-0.366,0.493]$} \\
\hline PEOU $\rightarrow$ ITUMAR & 0.769 & 14.636 & $* * *$ & 0.000 & {$[0.666,0.873]$} \\
\hline $\mathrm{PEOU} \rightarrow \mathrm{PU}$ & 0.742 & 9.096 & $* * *$ & 0.000 & {$[0.582,0.902]$} \\
\hline $\mathrm{UA} \rightarrow \mathrm{ITUMAR}$ & -0.145 & 0.851 & NS & 0.395 & {$[-0.480,0.190]$} \\
\hline $\mathrm{UA} \rightarrow \mathrm{PU}$ & -0.140 & 0.890 & NS & 0.374 & {$[-0.449,0.168]$} \\
\hline $\mathrm{UA} \rightarrow \mathrm{PEOU}$ & -0.189 & 0.873 & NS & 0.383 & {$[-0.613,0.235]$} \\
\hline
\end{tabular}

${ }^{* * *}$ Significance level of $5 \%$

NS: no significance.

TABLE 11: Cultural dimensions index.

\begin{tabular}{lcc}
\hline \multirow{2}{*}{ Member of an ethnic group } & \multicolumn{2}{c}{ Cultural factors } \\
& IDV & UA \\
\hline Yes & 44.50 & 87.50 \\
No & 12.91 & 63.45 \\
Total & 16.67 & 66.31 \\
\hline
\end{tabular}

a minimum of 70 observations for the sample size. Also, the minimum sample size was calculated with the approach called the rule of 10 times. The rule establishes that the minimum number of samples required for valid data can be obtained by multiplying by 10 times the maximum number of formative indicators of a latent variable. In this study, the latent variable with the highest number of indicators was PEOU with 8 indicators coming from the IDV and UA variables. Therefore, 8 multiplied by 10 results in the minimum required sample size of 80 . Based on the results obtained with the table of Hair (70 observations) and the rule of 10 times ( 80 observations), the asseveration that the data collected from 85 observations for this study was sufficient is supported.

A summary of the valid criteria used to perform the study is shown in Table 12.

It should be noted from Table 12 that the model proposed accomplishes all the accepted criteria; therefore, the interpretation of the results can be performed.
TABLE 12: Summary of accepted criteria for checking reliability and assessment of the proposed model.

\begin{tabular}{lc}
\hline $\begin{array}{l}\text { Reliability and assessment factors } \\
\text { of model }\end{array}$ & Accepted criterion \\
\hline$\alpha$ & Values between 0.6 and 1.0 \\
AVE & $>0.5$ \\
$\begin{array}{l}\text { Cross loadings of indicators with } \\
\text { their latent variables }\end{array}$ & $\begin{array}{c}\text { Higher values of the } \\
\text { indicators with their latent } \\
\text { variables }\end{array}$ \\
$\begin{array}{l}\text { Levels of collinearity } \\
\text { Outer weights }\end{array}$ & TOL $\geq 0.20$ and VIF $<5$ \\
Path coefficient relationships & Search for higher values \\
& Values between -1 and +1 \\
Path coefficient significance & Critical values $>t$
\end{tabular}

As can be observed in Figure 7, the path coefficient relationship of IDV contributes to PEOU with a positive 
value of 0.599 , and UA contributes with a value of -0.189 which is not statistically significant. According to Table 6, the indicators CF2 and PEOU3 have VIF values greater than 5 , which means that those indicators have critical levels of collinearity. However, the indicators were not removed from the survey because, in Table 8 , those indicators have a significant value.

The current research findings confirm that ITUMAR can be predicted by PU and PEOU, and PEOU is influenced by IDV and UA. The direct effects emphasize that PEOU and PU attained a strong positive direct influence on ITUMAR; therefore, hypotheses (H2) and (H3) are supported. PEOU is influenced positively by IDV; as a consequence, $(H 4)$ is supported. Also, it is important to mention from Table 9 the positive influence of IDV on PU and ITUMAR. The UA with a path coefficient near to zero has no positive effects on PEOU.

The outer weights computed in Table 8 for UA have no significance, and its index calculated for the students is high according to Table 11, which means that UA does not contribute to PEOU of the MAR system, and as a consequence hypothesis (H5) is rejected.

The variable PU has a negligible affectation path coefficient relationship of 0.063 on ITUMAR, and it is not significant for an error level of 5\%; therefore $(H 1)$ does not hold. This means that the influence of PU on ITUMAR is minimal or almost null.

Although ITUMAR2 indicator is not really an intention to use this particular system, its benefits were designed for the particular context of UAIM. The UAIM students are responsible for its learning; therefore, the learning activities must be performed even when the professor is absent. The essential parts of the MAR application such as the interaction and interface can be reused to design other learning activities.

The main variables with their coefficient relationships and the determination coefficients for accuracy prediction of the model are shown in Figure 7. The IDV and UA have no $R^{2}$ values because they are exogenous variables, which means that they are not caused by another variable in the model. ITUMAR have a $R^{2}$ value of 0.594 , PEOU equals 0.219 , and PU equals 0.550. Therefore, ITUMAR and PU are considered moderate and PEOU value is considered weak. The total effects between the variables are shown in Table 10. The relations between $\mathrm{PU} \rightarrow \mathrm{ITUMAR}, \mathrm{UA} \rightarrow \mathrm{ITUMAR}, \mathrm{UA} \rightarrow \mathrm{PU}$, and $\mathrm{UA} \rightarrow \mathrm{PEOU}$ are not statistically significant, which reaffirm the level of significance coefficients obtained in Table 7 and the outer weights of indicators obtained in Table 8 .

As can be observed in Table 11, the IDV variable has always low values $(<50)$ for all cases. Those values indicate that, in this study, students tend to be more of collectivists. Regarding UA, it should be noted that all values are high. Those values indicate that, in this study, students have great uncertainty of the unknown (new technology). This leads to the conclusion that the system could be used easily if it offers/promotes, in the future, a natural way for the collaborative work. For example, at least two users are working at the same time, on different devices, with the application. This will exploit the ability of the student to work in a collaborative way. In addition, the system could include help offered by other participants to have more trust in the system use and as a consequence reduce the UA of the student.

\section{Conclusions}

In this study, a novel approach to the assessment of the effects of the adoption of a MAR system for Mayo learning by undergraduates students was presented. The study was based on analyzing the interaction between the variables related to perception of cultural dimensions and variables related to the ITUMAR system. The application of the Lotería game was based on AR, where students interact directly with the educational content by relating images and audio. The application plays a recording message with the name of the object in the card to assist learning. The application was tested on mobile devices running Android operating system. According to the analysis of data collected from students after using the MAR system, the IDV as a cultural dimension has great influence on the ITUMAR system.

In the future, the goal will be to extend the study by increasing the number of undergraduates, creating subgroups by gender, age, and cultural differences to relate it with the ITU, and including the perceived enjoyment $(\mathrm{PE})$ variable. It will also be important to work with the system redesign to support collaborative or group work. This will lead to harnessing the positive elements related to IDV index of students. The consequence of promoting teamwork could be the reduction of the uncertainty that is presented when the student uses a new technology. Finally, because the indicators related to UA used in this study were treated as a social group type, it will be important to review these indicators for each individual. This could improve the accuracy of the measurements obtained.

\section{Appendix}

The questionnaire designed to obtain the information and validate the hypothesis is the following.

The Questionnaire. Please fill in the information about your general data (statistical purpose).

(SP1) Are you male or female?

(SP2) Do you belong to an ethnic group?

(SP3) How old are you?

Read the following statements and choose one of the following options for each statement: 1 Strongly disagree, 2 In disagreement 3 Somewhat disagree, 4 I do not know, 5 Somewhat agree, 6 In agreement, 7 Strongly agree.

\section{Perceived Usefulness}

(PU1) I could improve my learning performance by using the MAR system.

(PU2) I could enhance my language learning proficiency by using the MAR system.

(PU3) I could increase my learning productivity by using the MAR system. 
(PU4) I think that using the MAR system will helps me learn.

Perceived Ease of Use

(PEOU1) The design of the interface and the information provided by the MAR system is clear and easy to understand.

(PEOU2) It is easy to operate the MAR system.

(PEOU3) Overall I feel it is easy for me to use MAR system.

\section{Intention to Use the MAR}

(ITUMAR1) I will intend to use the MAR system for activities concerning the learning of Mayo language.

(ITUMAR2) The main principles of the system could be used for other learning activities.

Read the following statements and choose one of the following options for each statement: 1 Strongly unimportant, 2 Without unimportant, 3 Somewhat unimportant, 4 I do not know, 5 Somewhat important, 6 Important, 7 Very important.

\section{Cultural Factors}

Individualism

(CF1) Have sufficient time for your personal or home life.

(CF2) Have security of studying.

(CF3) The study is interesting.

(CF4) My family and my friends respected my studies.

Read the following statements and choose one of the following options for each statement: 1 Very poor, 2 Poor, 3 Little poor, 4 Fair, 5 Little good, 6 Good, 7 Very good.

\section{Uncertainty Avoidance}

(CF5) Generally, how would you describe your state of health these days?

Read the following statements and choose one of the following options for each statement: 1 Usually, 2 Sometimes, 3 Little, 4 I do not know, 5 It is rare, 6 Seldom, 7 Never.

(CF6) How often do you feel nervous or tense?

Read the following statements and choose one of the following options for each statement: 1 Strongly disagree, 2 In disagreement 3 Somewhat disagree, 4 I do not know, 5 Somewhat agree, 6 In agreement, 7 Strongly agree.

(CF7) A person can be a good teacher even without knowing the answer to the question submitted to it relating to their work.

(CF8) The rules dictated by the school should never be broken, not even for the best interests of the school.

\section{Competing Interests}

The authors declare that they have no competing interests.

\section{References}

[1] A. Surian, "Aprendizaje y competencias culturales," Ra-Ximhai, vol. 8, no. 2, pp. 205-222, 2012.

[2] H. P. Chia and A. Pritchard, "Using a virtual learning community (VLC) to facilitate a cross-national science research collaboration between secondary school students," Computers and Education, vol. 79, pp. 1-15, 2014.

[3] R. House, P. Hanges, M. Javidan, P. Dorfman, and V. Gupta, Culture, Leadership, and Organizations: The GLOBE Study of 62 Societies, Sage Publications, 1st edition, 2004.

[4] G. Hofstede, G. J. Hofstede, and M. Minkov, Cultures and Organizations: Software of the Mind, McGraw-Hill, New York, NY, USA, 3rd edition, 2010.

[5] F. Trompenaars and C. Hampden Turner, Riding the Waves of Culture: Understanding Cultural Diversity in Business, Nicholas Brealey Publishing, 5th edition, 2011.

[6] V. Taras, P. Steel, and B. L. Kirkman, "Improving national cultural indices using a longitudinal meta-analysis of Hofstede's dimensions," Journal of World Business, vol. 47, no. 3, pp. 329341, 2012.

[7] D. Potnis, "Culture's consequences: economic barriers to owning mobile phones experienced by women in India," Telematics and Informatics, vol. 33, no. 2, pp. 356-369, 2016.

[8] A. I. Khan, H. Al-Shihi, Z. A. Al-Khanjari, and M. Sarrab, "Mobile Learning (M-Learning) adoption in the Middle East: lessons learned from the educationally advanced countries," Telematics and Informatics, vol. 32, no. 4, pp. 909-920, 2015.

[9] I. Reychav, M. Dunaway, and M. Kobayashi, "Understanding mobile technology-fit behaviors outside the classroom," Computers and Education, vol. 87, article 2836, pp. 142-150, 2015.

[10] M. Kearney, K. Burden, and T. Rai, "Investigating teachers' adoption of signature mobile pedagogies," Computers \& Education, vol. 80, pp. 48-57, 2015.

[11] L. Johnson, R. Smith, H. Willis, A. Levine, and K. Haywood, "The 2011 horizon report," Tech. Rep., The New Media Consortium, Austin, Tex, USA, 2011.

[12] J. Martín-Gutiérrez, P. Fabiani, W. Benesova, M. D. Meneses, and C. E. Mora, "Augmented reality to promote collaborative and autonomous learning in higher education," Computers in Human Behavior, vol. 51, pp. 752-761, 2015.

[13] R. T. Azuma, "A survey of augmented reality," Presence: Teleoperators and Virtual Environments, vol. 6, no. 4, pp. 355-385, 1997.

[14] N. S. Baron and E. M. Campbell, "Gender and mobile phones in cross-national context," Language Sciences, vol. 34, no. 1, pp. 13-27, 2012.

[15] S.-G. Lee, S. Trimi, and C. Kim, "The impact of cultural differences on technology adoption," Journal of World Business, vol. 48, no. 1, pp. 20-29, 2013.

[16] O. Viberg and Å. Grönlund, "Cross-cultural analysis of users' attitudes toward the use of mobile devices in second and foreign language learning in higher education: a case from Sweden and China," Computers and Education, vol. 69, pp. 169-180, 2013.

[17] M. M. Al-Debei and E. Al-Lozi, "Explaining and predicting the adoption intention of mobile data services: a value-based approach," Computers in Human Behavior, vol. 35, no. 1, pp. 326-338, 2014. 
[18] G. Baptista and T. Oliveira, "Understanding mobile banking: the unified theory of acceptance and use of technology combined with cultural moderators," Computers in Human Behavior, vol. 50, pp. 418-430, 2015.

[19] K. Lee, "Augmented reality in education and training," TechTrends, vol. 56, no. 2, pp. 13-21, 2012.

[20] M. Billinghurst and A. Dünser, "Augmented reality in the classroom," Computer, vol. 45, no. 7, Article ID 6171143, pp. 5663, 2012.

[21] H.-K. Wu, S. W.-Y. Lee, H.-Y. Chang, and J.-C. Liang, "Current status, opportunities and challenges of augmented reality in education," Computers \& Education, vol. 62, pp. 41-49, 2013.

[22] E. Klopfer and J. Sheldon, "Augmenting your own reality: student authoring of science-based augmented reality games," New Directions for Youth Development, vol. 2010, no. 128, pp. 85-94, 2010.

[23] F. Ke and Y.-C. Hsu, "Mobile augmented-reality artifact creation as a component of mobile computer-supported collaborative learning," The Internet and Higher Education, vol. 26, pp. 33-41, 2015.

[24] M. Dunleavy and C. Dede, "Handbook of research on educational communications and technology," in Augmented Reality Teaching and Learning, pp. 735-745, Springer, New York, NY, USA, 2014.

[25] J. Cheon, S. Lee, S. M. Crooks, and J. Song, "An investigation of mobile learning readiness in higher education based on the theory of planned behavior," Computers and Education, vol. 59, no. 3, pp. 1054-1064, 2012.

[26] F. D. Davis, "Perceived usefulness, perceived ease of use, and user acceptance of information technology," MIS Quarterly, vol. 13, no. 3, pp. 319-339, 1989.

[27] N. Marangunić and A. Granić, “Technology acceptance model: a literature review from 1986 to 2013," Universal Access in the Information Society, vol. 14, no. 1, pp. 81-95, 2015.

[28] F. D. Davis, R. P. Bagozzi, and P. R. Warshaw, "User acceptance of computer technology: a comparison of two theoretical models," Management Science, vol. 35, no. 8, pp. 982-1003, 1989.

[29] I.-F. Liu, M. C. Chen, Y. S. Sun, D. Wible, and C.-H. Kuo, "Extending the TAM model to explore the factors that affect intention to use an online learning community," Computers \& Education, vol. 54, no. 2, pp. 600-610, 2010.

[30] A. Padilla-Meléndez, A. R. Del Aguila-Obra, and A. GarridoMoreno, "Perceived playfulness, gender differences and technology acceptance model in a blended learning scenario," Computers and Education, vol. 63, pp. 306-317, 2013.

[31] Y. Liu, H. Li, and C. Carlsson, "Factors driving the adoption of m-learning: An Empirical Study," Computers and Education, vol. 55, no. 3, pp. 1211-1219, 2010.

[32] T.-P. Liang and Y.-H. Yeh, "Effect of use contexts on the continuous use of mobile services: the case of mobile games," Personal and Ubiquitous Computing, vol. 15, no. 2, pp. 187-196, 2011.

[33] A.-C. Haugstvedt and J. Krogstie, "Mobile augmented reality for cultural heritage: a technology acceptance study," in Proceedings of the 11th IEEE International Symposium on Mixed and Augmented Reality (ISMAR '12), pp. 247-255, IEEE, Atlanta, Ga, USA, November 2012.

[34] M. N. Giannakos, "Enjoy and learn with educational games: examining factors affecting learning performance," Computers and Education, vol. 68, pp. 429-439, 2013.
[35] R. Wojciechowski and W. Cellary, "Evaluation of learners' attitude toward learning in ARIES augmented reality environments," Computers and Education, vol. 68, pp. 570-585, 2013.

[36] J. Boase and R. Ling, "Measuring mobile phone use: self-report versus log data," Journal of Computer-Mediated Communication, vol. 18, no. 4, pp. 508-519, 2013.

[37] J. Arenas-Gaitán, P. E. Ramírez-Correa, and F. Javier RondánCataluña, "Cross cultural analysis of the use and perceptions of web Based learning systems," Computers and Education, vol. 57, no. 2, pp. 1762-1774, 2011.

[38] G. Hofstede, "Values survey measure (VSM-2013)," 2013, http:// www.geerthofstede.nl/vsm2013.

[39] A. R. Montazemi and H. Qahri-Saremi, "Factors affecting adoption of online banking: a meta-analytic structural equation modeling study," Information \& Management, vol. 52, no. 2, pp. 210-226, 2015.

[40] J. C. Cronjé, "Using Hofstede’s cultural dimensions to interpret cross-cultural blended teaching and learning," Computers and Education, vol. 56, no. 3, pp. 596-603, 2011.

[41] J. Hair, G. Hult, C. Ringle, and M. Sarstedt, A Primer on Partial Least Squares Structural Equation Modeling (PLS-SEM), Sage, Los Angeles, Calif, USA, 1st edition, 2014.

[42] J. Henseler, C. M. Ringle, and M. Sarstedt, "A new criterion for assessing discriminant validity in variance-based structural equation modeling," Journal of the Academy of Marketing Science, vol. 43, no. 1, pp. 115-135, 2014.

[43] W. Reinartz, M. Haenlein, and J. Henseler, "An empirical comparison of the efficacy of covariance-based and variancebased SEM," International Journal of Research in Marketing, vol. 26, no. 4, pp. 332-344, 2009.

[44] D. L. Goodhue, W. Lewis, and R. Thompson, "Does pls have advantages for small sample size or non-normal data?" MIS Quarterly: Management Information Systems, vol. 36, no. 3, pp. 981-1001, 2012.

[45] J. Cohen, Statistical Power Analysis for the Behavioral Sciences, Lawrence Erlbaum Associates, New York, NY, USA, 2nd edition, 1998. 

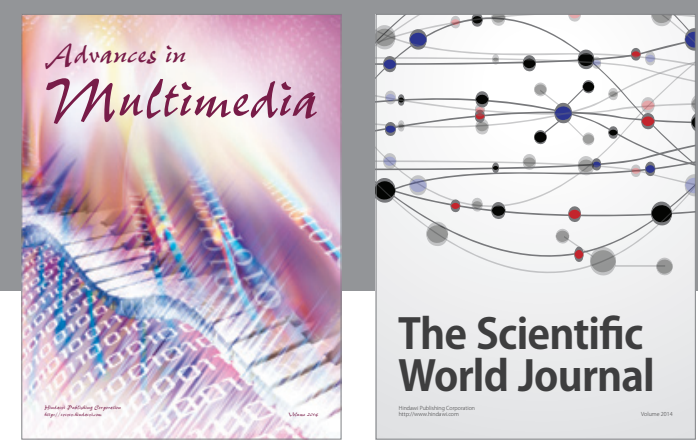

The Scientific World Journal
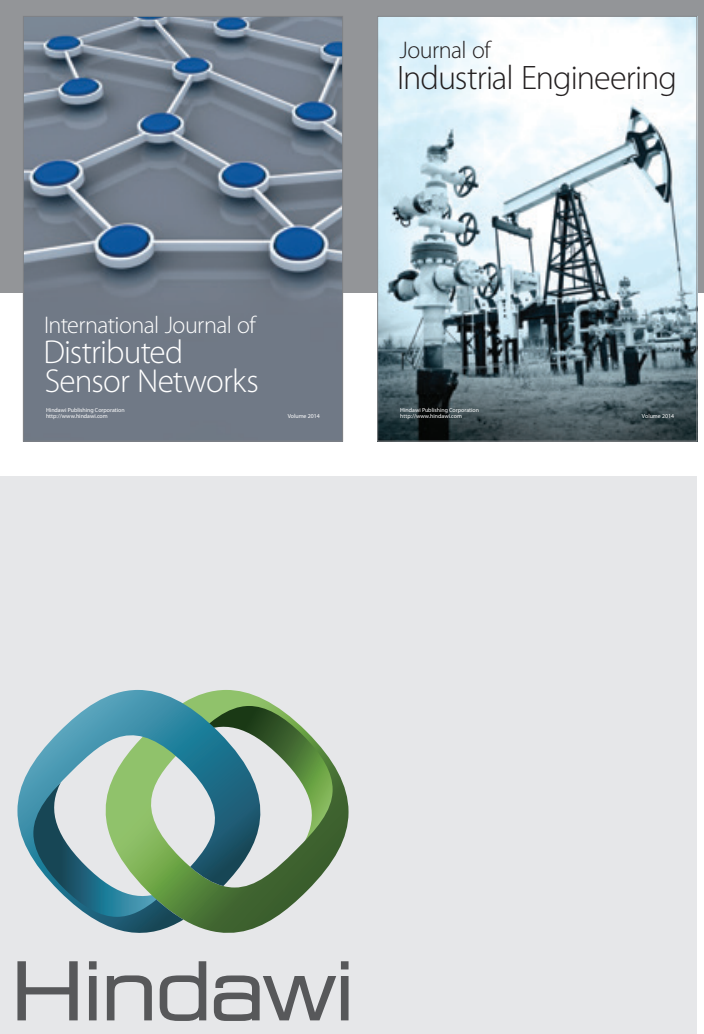

Submit your manuscripts at

http://www.hindawi.com

\section{Computer Networks} and Communications
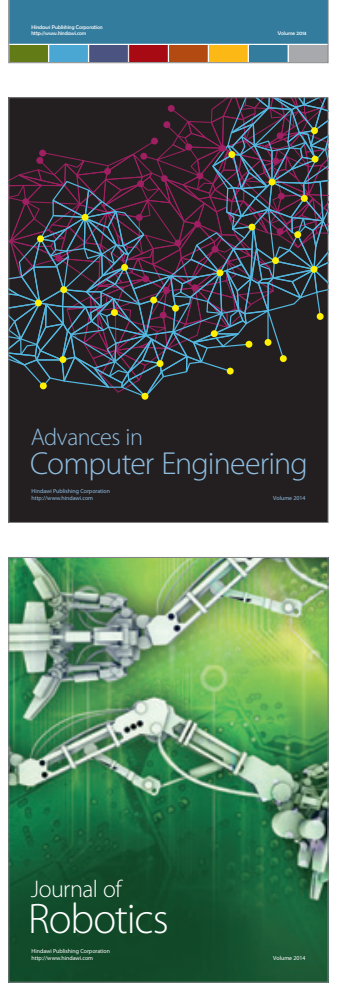
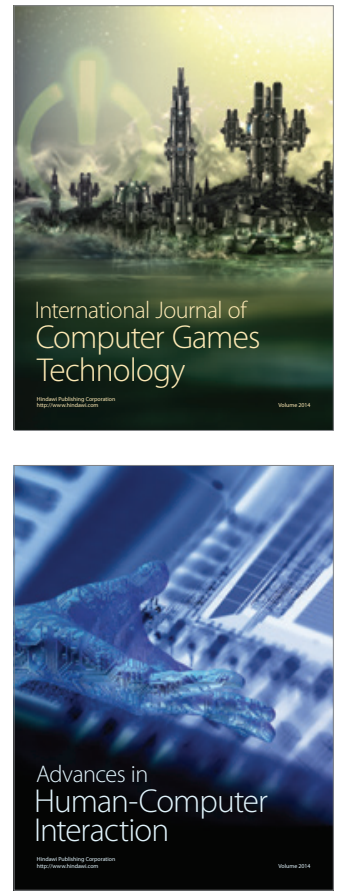
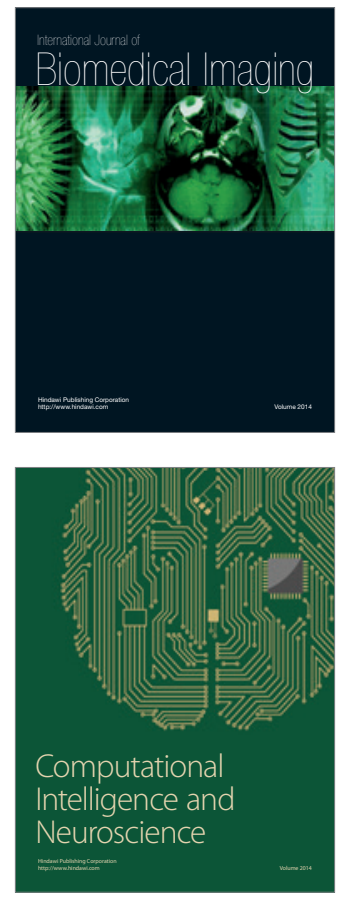
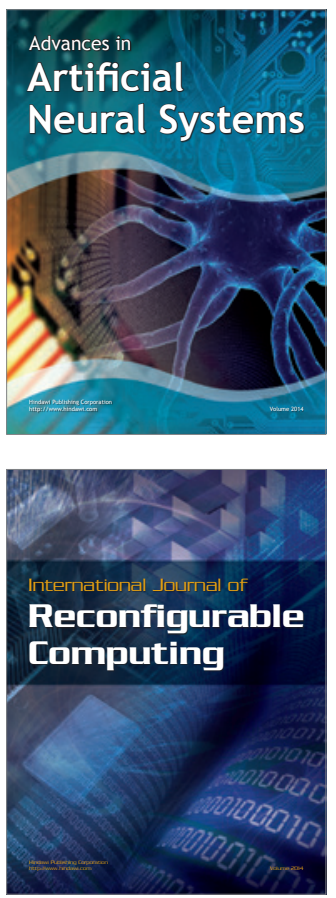
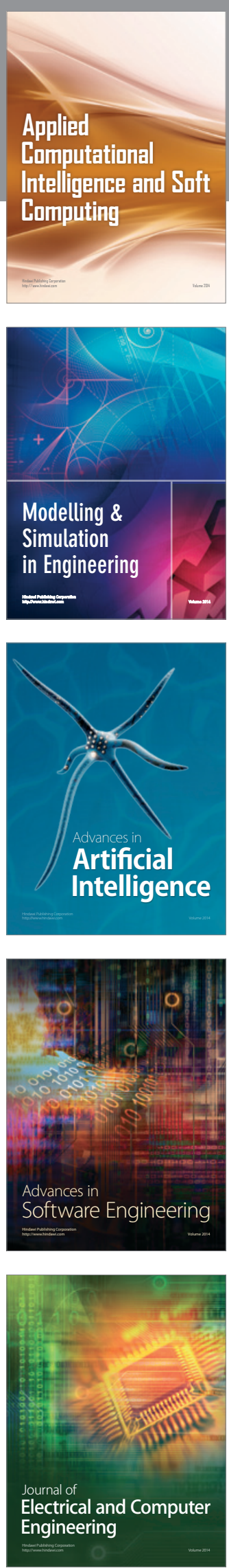